\title{
Electrostatic Subframing and Compressive Sensing Video in In Situ Transmission Electron Microscopy
}

\author{
B. W. Reed, ${ }^{1 *}$ A. A. Moghadam, ${ }^{1}$ R. S. Bloom, ${ }^{1}$ S. T. Park, ${ }^{1}$ A. M. Monterrosa, ${ }^{2}$ P. M. Price,${ }^{2}$ C. M. \\ Barr, ${ }^{2}$ S. A. Briggs, ${ }^{2,3}$ K. Hattar, ${ }^{2}$ and D. J. Masiel ${ }^{1}$ \\ 1. Integrated Dynamic Electron Solutions, Pleasanton, CA, USA \\ 2. Sandia National Laboratories, Albuquerque, NM 87123, USA \\ 3. Oregon State University, Corvallis, OR 97331, USA \\ * Corresponding author: bryan@ides-inc.com
}

Recently we have been developing a high-speed transmission electron microscope (TEM) video acquisition system based on electrostatic subframing (ES), which uses a fast post-sample deflector to array multiple images across a large TEM camera (Figure 1).[1] Originally designed for compressivesensing (CS) in situ burst-mode video acquisition at $\mathrm{kHz}$ frame rates, we have found the system capable of multiple operating modes including many not envisioned in its original design. Ultimately, ES is a system for both extending and enhancing a camera's pixel-count/frame-rate tradeoff curve well beyond its usual range, in both burst and continuous acquisition modes (Figure 2).

ES extends the tradeoff curve by direct subdivision of the camera area into a $2 \times 2,3 \times 3,4 \times 4$, or even $16 \times 16$ array of subframes. This allows, for example, a $4 \mathrm{k} \times 4 \mathrm{k}$ pixel camera to act as four parallel, rapidly selectable $2 \mathrm{k}$ x 2k pixel cameras; or $161 \mathrm{k}$ x 1k cameras; or $256256 \times 256$ pixel cameras; or many steps in between. In continuous-acquisition mode, this allows a 10-100 Hz camera to act as a kHz-scale camera with fewer pixels and $100 \%$ duty cycle, while in burst mode it allows even a sub-Hz camera to reach multi-kHz frame rates. Both modes enable in situ experiments that would be otherwise impossible on a given camera.

ES enhances the tradeoff curve by using CS and related sparsity-based reconstruction techniques to take advantage of both spatial overlaps of subframes on the camera and temporal overlaps of pseudorandomly-undersampled video frames. Thus, the pixel-count/frame-rate tradeoff curve can be better than 1-to-1; the reconstructed video from a single exposure can have more space-time voxels than the actual camera has pixels, by factors of 6 or more in tests to date. This means that a $4 \mathrm{k} x 4 \mathrm{k}$ pixel camera can, in a single exposure, produce not just $16>1$-megapixel images but 100 of them, with frame rates of multiple $\mathrm{kHz}$. This has numerous proven and potential applications including in situ TEM, 4DSTEM, and high-throughput tomography.

References:

[1] B. W. Reed et al., Microsc. Microanal. 24 (suppl. 1), 494 (2018).

[2] This material is based upon work supported by the U.S. Department of Energy, Office of Science, Office of Basic Energy Sciences, under Award Number DE-SC0013104. This work was performed, in part, at the Center for Integrated Nanotechnologies, an Office of Science User Facility operated for the U.S. Department of Energy (DOE) Office of Science. Sandia National Laboratories is a multi-mission laboratory managed and operated by National Technology and Engineering Solutions of Sandia, LLC., a wholly owned subsidiary of Honeywell International, Inc., for the U.S. DOE's National Nuclear Security Administration under contract DE-NA-0003525. The views expressed in the article do not 
necessarily represent the views of the U.S. DOE or the United States Government. Document SAND2019-1905 C

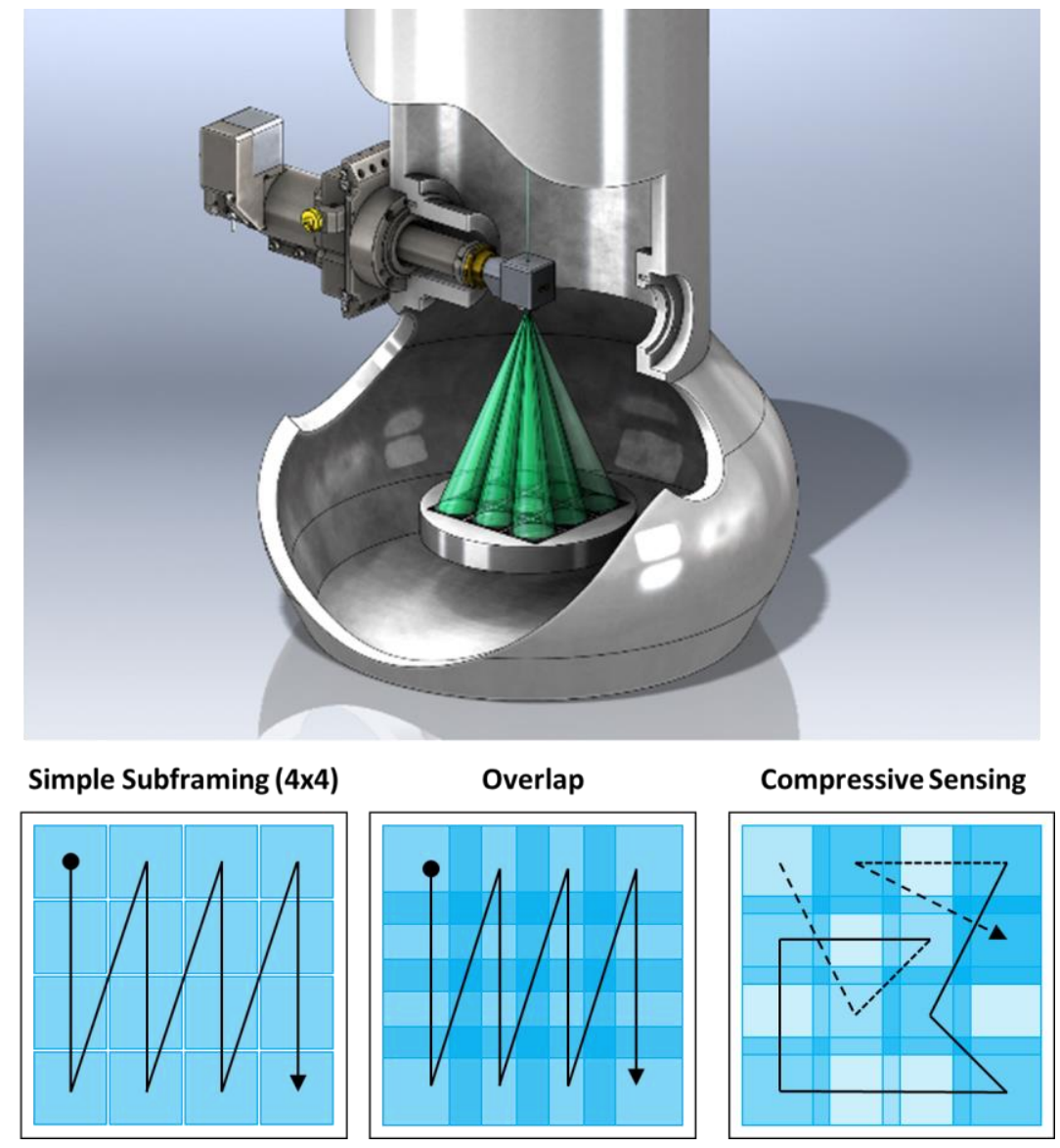

Figure 1. The electrostatic subframing system with schematic illustrations of its primary operating modes, using a $4 \times 4$ array of subframes: Simple subframing, subdividing the camera into 16 regions; Subframing with overlap, using CS reconstruction algorithms to determine which features in the overlap regions belong in each subframe; and full CS mode, in which the subframes are sequenced in an arbitrary pseudorandom order during each camera exposure, allowing for example 100 frames of video to be reconstructed from only 16 subframes.

Figure 2. Advantages of ES for improving the frame-rate/pixel-count tradeoff curve far beyond what is achievable with binning alone. Example plotted for a 16-megapixel camera, assuming 20\% subframe overlap on each interior edge and 6:1 video data compression ratio in $\mathrm{CS}$ mode. We have plotted sustained frame rate enhancement factors, e.g. a $1 \mathrm{~Hz}$ camera in 16x16 mode in full CS/overlap mode should produce over 1000 frames per second with over 100,000 pixels per frame. Burst mode rates can be even higher - up to $10 \mathrm{kHz}$ in tests to date using a conventional sub-Hz camera.

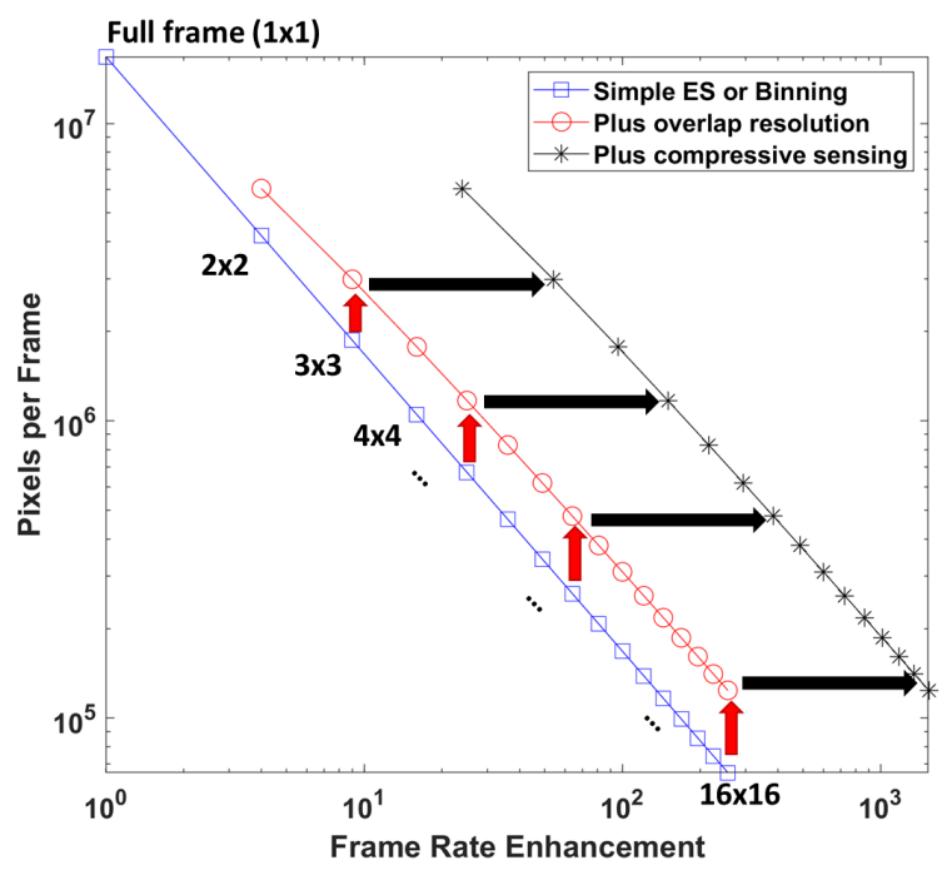

